\title{
Ultrafast pulse generation in semiconductor lasers
}

\author{
Marc Sorel \\ School of Engineering \\ University of Glasgow \\ Glasgow, G12 8LT \\ United Kingdom
}

\author{
Michael J. Strain \\ Institute of Photonics \\ Dept. of Physcis \\ University of Strathclyde \\ Glasgow, G1 1RD \\ United Kingdom \\ Email: michael.strain@strath.ac.uk
}

\begin{abstract}
Integrated semiconductor laser devices are presented as extremely compact generators of ultra-short pulse trains. Control is demonstrated on a wide range of emission parameters including wavelength, pulse duration, repetition rate and emitted power. All device geometries require simple drive electronics, consisting of only constant current injection and reverse bias voltage control.
\end{abstract}

\section{INTRODUCTION}

Ultrashort optical pulses are used in a wide variety of applications from telecommunications to $3 D$ imaging. Often it is advantageous for the source of such short pulses to be compact, mechanically robust and exhibit low power consumption. Integrated, planar semiconductor lasers are an attractive technology in these circumstances due to their monolithic integration, small size and potential for integration with simple board level drive electronics. However, with integrated laser cavities, exercising control over the laser pulse characteristics is not as direct a process as for free-space laser systems where elements can be easily added, removed or modified within the cavity. Nevertheless, with careful optical design of the onchip laser structure the full range of optical pulse features can be accessed. These include, pulse-duration, average and peak power [1], dispersion [2], emission wavelength [3] and repetition rate [4], [5]. By using the toolbox of on-chip photonic components available, all of these laser characteristics can be designed to suit the application and even exhibit an amount of active control.

In this work a range of integrated semiconductor lasers are presented illustrating the optimisation of the on-chip cavity designs for control of each of the pulse characteristics listed above. Most of the lasers are modifications on the now standard mode-locked laser (MLL) cavity design, incorporating a gain section, a saturable absorber, and cavity reflectors. In all of the devices a significant design specification is to maintain their compact footprint whilst introducing the necessary control elements to the device to allow the modulation of the above listed pulse parameters.

\section{BASIC SEMICONDUCTOR LASER DESIGNS}

The simplest integrated semiconductor mode-locked laser (MLL) design, shown in Fig.1, is easy to fabricate and requires only continuous wave current injection and reverse bias voltage signals to be applied to generate ultrashort pulses.
Even with such simplicity, pulse durations of a few hundred femtoseconds have been demonstrated using these Fabry-Perot (FP) type devices [6]. Fig.2 shows a measured optical spectrum

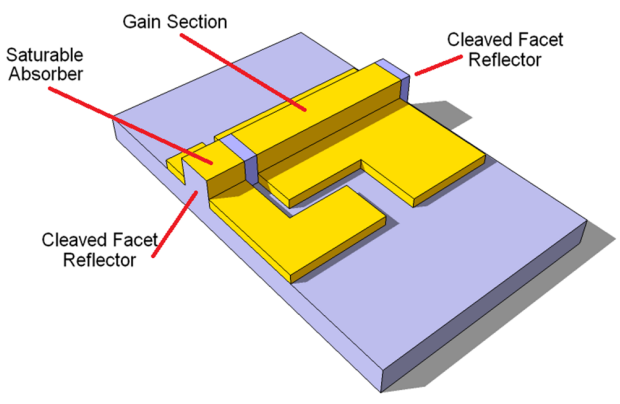

Fig. 1. Schematic of simple Fabry-Perot semiconductor mode-locked laser.

and intensity autocorrelation trace for a FP MLL device [11]. The trade-off for the compact and simple device geometry is apparent in the operating parameter space of the laser. The relationships between the laser emission wavelength, repetition rate and pulse duration as a function of injection conditions will be described in detail. It will also be made clear that the operating characteristics of the lasers are strongly interlinked, so that active control over one aspect has inherent effects on others. This presentation will detail the various laser devices that have been developed in order to optimise a particular aspect of the emission regime.

\section{SPECTRAL AND POWER CONTROL}

Inclusion of a spectrally selective element in the laser cavity has a number of effects on the laser operation. This filter is easily incorporated into the FP MLL design by replacing one of the cleaved mirrors by a Bragg grating filter [3]. Fig.3 shows the modified schematic of the FP MLL including a spectrally selective Bragg filter. The Bragg condition of the filter is defined by the period of the grating, and this in turn defines the emission wavelength of the laser. Fig.4 shows measured spectra of Bragg grating filters with varying period, spanning the gain bandwidth of a quantum-well laser material system.

As predicted by [7], the selection of the emission wavelength by an intra-cavity filter has a strong effect on the laser parameters, in particular the injection current and reverse bias voltage ranges over which the laser will operate in the 

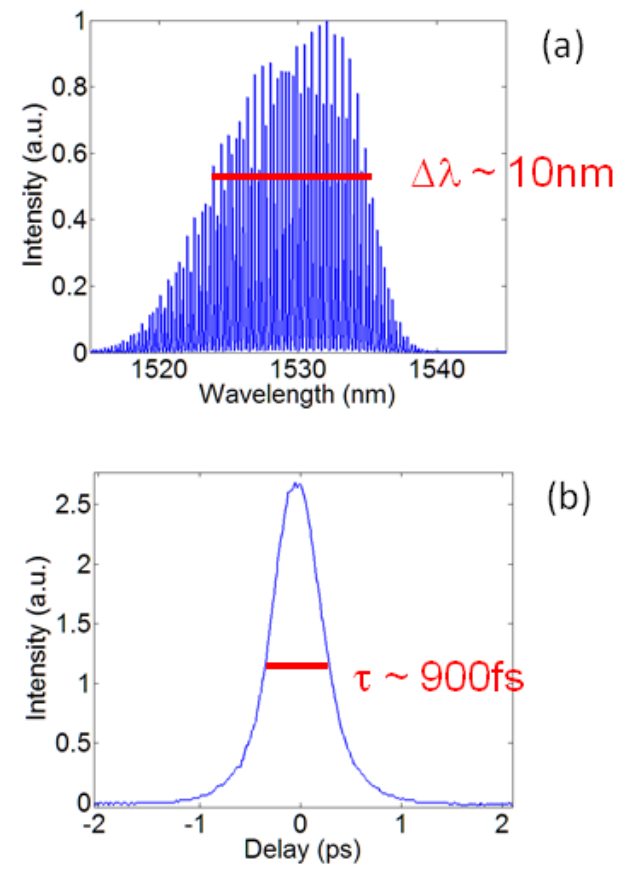

(b)

Fig. 2. (a) Measured optical spectrum and (b) intensity autocorrelation of a FP semiconductor MLL.

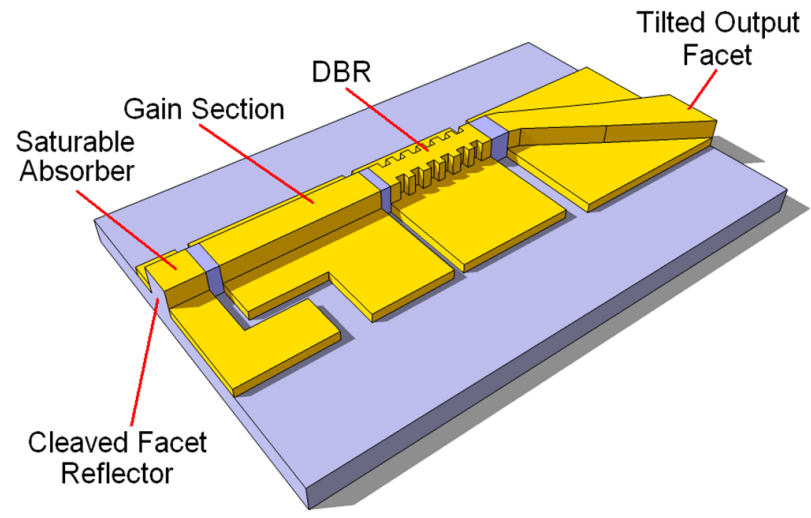

Fig. 3. Schematic of Fabry-Perot semiconductor mode-locked laser with an intra-cavity Bragg mirror section.

mode-locked regime. By local control over the intra-cavity filter tempro-spectral function, control can also be gained over the ultrashort pulse chirp behaviour. Furthermore, individual reflectors can be multiplexed in a single laser device, allowing locking of sub-bands to a single mode-locked pulse train [3].

By using on-chip reflectors, extra-cavity amplifier sections can be monolithically integrated with the MLL devices, allowing further control over their average power levels, without degrading temporal pulse durations [1].

Alternatively, post-fabrication modification of the saturable absorber band-edge through intermixing techniques [8], modifies the laser emission wavelength and hence operating regime as a function of injection conditions. This method although less spectrally selective than using intra-cavity filters maintains

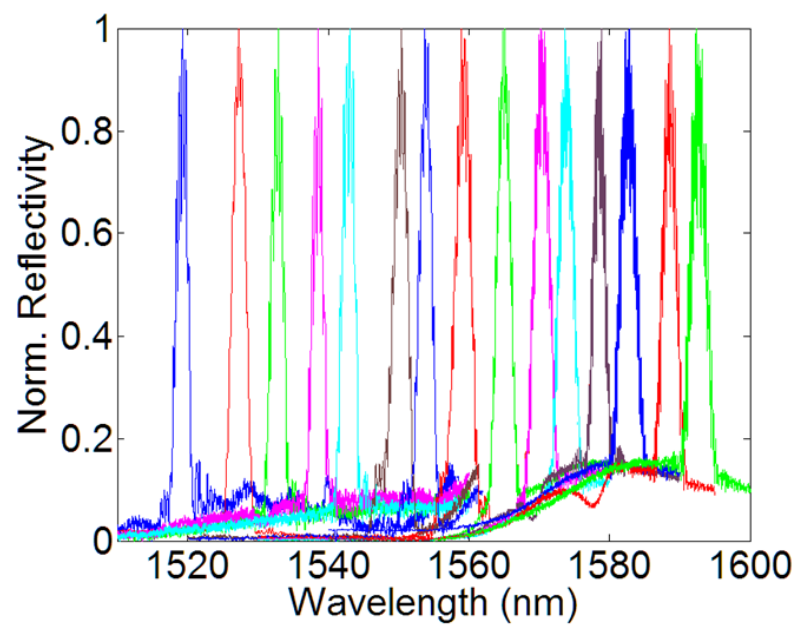

Fig. 4. Measured integrated Bragg grating filter responses in FP MLL geometries.

the broad laser bandwidth and sub-ps pulse durations.

\section{REPETITION RATE}

In simple FP or ring type semiconductor MLLs the repetition rate of the ultrashort pulse train is simply defined by the round-trip time of the cavity. This limits any control over the repetition rate post-fabrication to small variations related to the electrical injection modulation of the material refractive index. There are however other possible geometries to allow both higher and lower rates than the geometric round-trip. Higherharmonic (HH) mode-locking has been demonstrated using saturable absorbers at sub-cavity lengths within the laser [4], [9]. This technique allows the generation of repetition rates that are multiples of the fundamental round-trip, and different operating points can be selected post-fabrication by using more than one saturable absorber within the laser, Fig.5.

For low repetition rates there are two main options. The first involves creating long folded cavity geometries on-chip to increase the pulse round-trip time [10]. The second uses an intra-cavity filter to change the operating regime of the laser [5]. The interplay of the absorber bandedge, gain peak and injection conditions can force the laser to operate in the simple mode-locked regime, or as a hybrid Q-switched MLL, as shown in Fig.6. In this latter regime the pulse repetition rate can be lowered into the few $\mathrm{GHz}$ regime, with the associated increase in peak power and a few GHz tunability.

\section{Alternative device geometries}

It is possible to create stable pulse trains in semiconductor lasers without the use of a saturable absorber in the cavity. Different geometries for stabilised mode beating in semiconductor lasers will be presented with tunability of the beat frequency extending over the tens of $\mathrm{GHz}$ range. These devices again do not require any high speed drive electronics to generate stable frequencies in the few to hundreds of $\mathrm{GHz}$ range. 


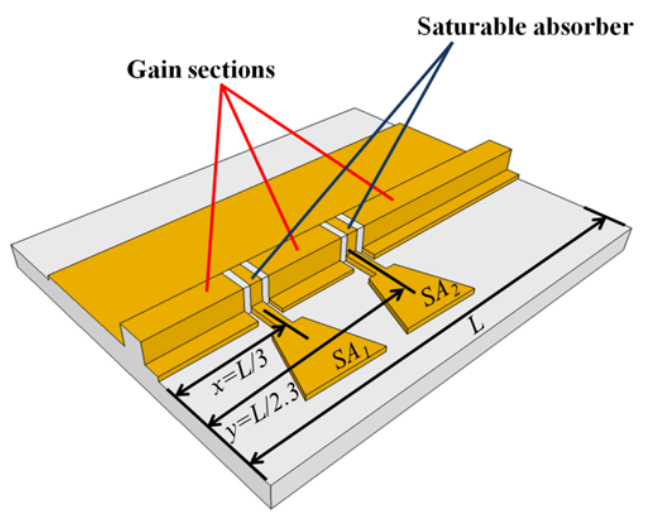

$\mathrm{M}=2, \quad 70 \mathrm{GHz}$
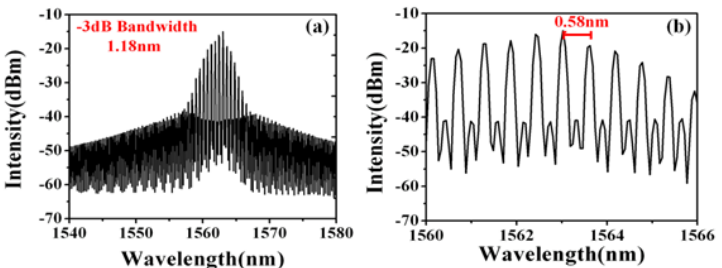

$\mathrm{M}=3, \quad 105 \mathrm{GHz}$
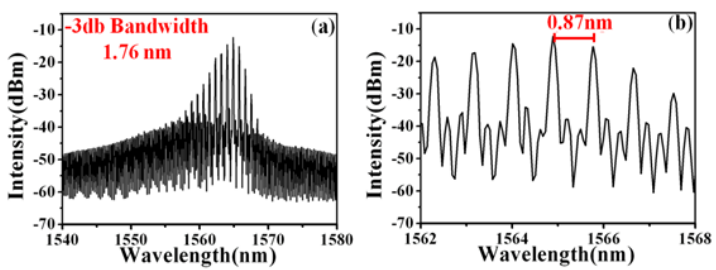

Fig. 5. Schematic of a multi-section HH MLL and measured spectral characteristics at two different harmonic ratios.

\section{CONCLUSION}

On-chip semiconductor lasers have been demonstrated with a wide range of operating conditions, relying on simple drive electronics and various cavity geometries. Control over the generated pulse duration, repetition rate and emission wavelength have been exhibited along with the inherent interconnectedness of these parameters.

\section{ACKNOWLEDGMENT}

The authors would like to thank the staff of the James Watt Nanofabrication Centre at the University of Glasgow. This work was partially supported by the EPSRC (EP/E065112/1).

\section{REFERENCES}

[1] J. Akbar, L. Hou, M. Haji, M. J. Strain, P. M. Stolarz, J. H. Marsh, A. C. Bryce, and A. E. Kelly, High peak power $(550 \mathrm{~mW}) 40 \mathrm{GHz}$ mode-locked DBR lasers with integrated optical amplifiers, in Photonics Conference (PHO), 2011 IEEE, 2011, pp. 755756.

[2] M. J. Strain, P. M. Stolarz, and M. Sorel, Passively mode-locked lasers with integrated chirped bragg grating reflectors, Quantum Electron. IEEE J., vol. 47, no. 4, pp. 492499, 2011.

[3] M. J. Strain, M. Zanola, G. Mezosi, and M. Sorel, Generation of Picosecond Pulses Over a 40-nm Wavelength Range Using an Array of Distributed Bragg Grating Mode-Locked Lasers, IEEE Photonics Technol. Lett., vol. 25, no. 4, pp. 368370, Feb. 2013.
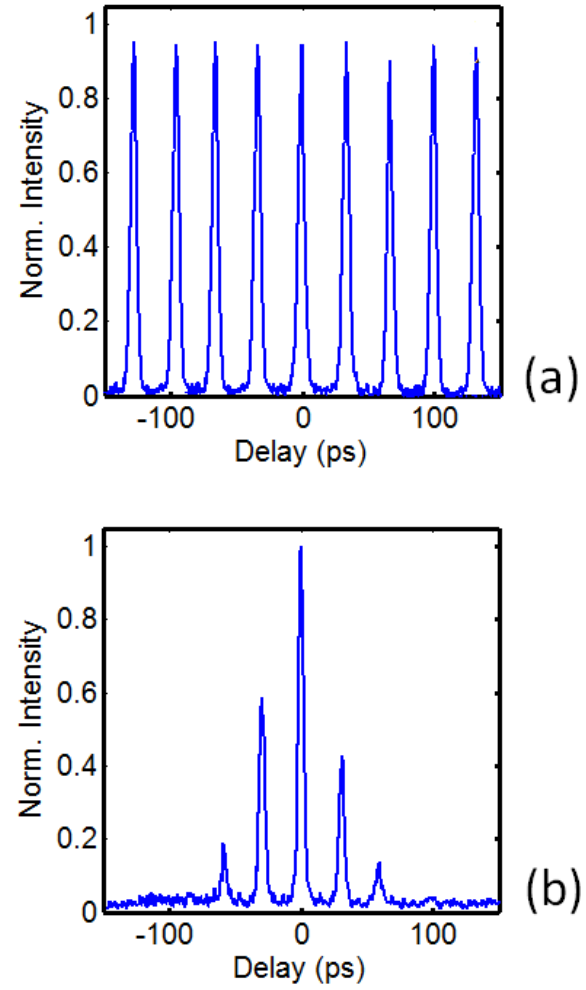

Fig. 6. Autocorrelation traces for a MLL operating in the (a) simple modelocking and (b) hybrid Q-switched mode-locking regimes.

[4] A. R. Rae, M. G. Thompson, R. V. Penty, I. H. White, a. R. Kovsh, S. S. Mikhrin, D. a. Livshits, and I. L. Krestnikov, Harmonic Mode-Locking of a Quantum-Dot Laser Diode, 2006 IEEE LEOS Annu. Meet. Conf. Proc., pp. 874875, Oct. 2006.

[5] M. J. Strain, M. Zanola, G. Mezsi, and M. Sorel, Ultrashort Q-switched pulses from a passively mode-locked distributed Bragg reflector semiconductor laser, Opt. Lett., vol. 37, no. 22, pp. 47324734, 2012.

[6] Z. G. Lu, J. R. Liu, S. Raymond, P. J. Poole, P. J. Barrios, and D. Poitras, 312-fs pulse generation from a passive C-band InAs/InP quantum dot mode-locked laser., Opt. Express, vol. 16, no. 14, pp. 1083540, Jul. 2008.

[7] P. M. Stolarz, J. Javaloyes, G. Mezosi, L. Hou, C. N. Ironside, M. Sorel, a. C. Bryce, and S. Balle, Spectral Dynamical Behavior in Passively Mode-Locked Semiconductor Lasers, IEEE Photonics J., vol. 3, no. 6, pp. 10671082, Dec. 2011.

[8] V. Pusino, M. J. Strain, and M. Sorel, Passive mode-locking in semiconductor lasers with saturable absorbers bandgap shifted through quantum well intermixing, vol. 2, no. 6, pp. 186189, 2014.

[9] Shahid Mahmood, M. Haji, Philip. J. Poole, A.Catrina Bryce, M. Sorel and Michael J. Strain, High Harmonic Mode-locked InP Quantum Dot Lasers in European Semiconductor Laser Workshop, 23-24 September 2011, Lausanne, Switzerland.

[10] S. Srinivasan, S. Member, A. Arrighi, M. J. R. Heck, J. Hutchinson, E. Norberg, G. Fish, and J. E. Bowers, Harmonically Mode-Locked Hybrid Silicon Laser With Intra-Cavity Filter to Suppress Supermode Noise, IEEE J. Sel. Top. Quantum Electron., vol. 20, no. 4, 2014.

[11] P. M. Stolarz, G. Mezosi, M. J. Strain, A. C. Bryce, and M. Sorel, Highly-sensitive sonogram for assessment of chirp in semiconductor mode-locked lasers, Quantum Electron. IEEE J., vol. 48, no. 8, pp. 9951003, 2012 